Check for updates

Cite this: Digital Discovery, 2022, 1, 6

\title{
A forward view for Digital Discovery: the scientific challenges of the twenty-first century require accelerated discovery approaches
}

\author{
Alán Aspuru-Guzikł ${ }^{\mathrm{abc}}$
}

DOI: $10.1039 / \mathrm{d} 2 \mathrm{dd} 90001 \mathrm{~g}$

rsc.li/digitaldiscovery

The inaugural editorial of a new journal is an excellent opportunity to set the expectations for the contents to be included in its (digital) issues-to-come, especially when the journal's scope covers an emerging area of research. For example, on January 1 of 1933 , in the opening editorial of the Journal of Chemical Physics ${ }^{1}$ Harold C. Urey aimed to introduce and embrace the new field of Chemical Physics at the interface of chemistry and physics by describing it as follows: "the methods of investigation used are, to a large extent, not those of classical chemistry, and the field is not of primary interest to the main body of physicists, nor is it the traditional field of physics. It seems proper that a journal devoted to this borderline field should be available to this group". Additionally, more recently in the book entitled Neither Physics nor Chemistry, ${ }^{2}$ two science historians, Kostas and Gavroglu, argued for the birth of Quantum Chemistry as a new field at the interface of physics, chemistry, applied mathematics, and what is now considered computer science.

\footnotetext{
${ }^{a}$ Professor of Chemistry, Computer Science, and Chemical Engineering and Applied Chemistry, University of Toronto, Toronto, Ontario, M5S 2E4, Canada

${ }^{b}$ CIFAR AI Chair, Vector Institute, Toronto, Ontario, M5S 1M1, Canada

${ }^{c}$ CIFAR Lebovic Fellow, Toronto, Ontario, M5G 1Z8, Canada.E-mail: alan@aspuru.com

$\dagger$ Editor-in-Chief, Digital Discovery.
}

Likewise, this inaugural editorial argues that we are currently at an analogous junction observing the birth of a new field and, therefore, a new journal is needed to fill this gap.

One can argue that there are similarities and differences between 1933, when Journal of Chemical Physics was launched, and today. In 1933, the world was emerging from a pandemic that ten years earlier purged the global population and was facing the rise of authoritarian regimes worldwide. I believe we share a modern reenactment of those challenges in the current decade. On the other hand, unlike in the early $20^{\text {th }}$ century, today we also face substantial ecological challenges related to climate change, rapid deforestation, pollution, new diseases, and an ageing population.

In a relatively recent perspective published in ACS Central Science, Roland Lindh, Markus Reiher and $\mathrm{I}^{3}$ discussed what we considered to be the challenges for computational science and computational and theoretical chemistry to match the societal needs present in the twentyfirst century. We concluded that chemistry (both theoretical and experimental) needs to further interface with the twenty-first century's technological driving fields to address the prescient challenges briefly mentioned above. The technological drivers that we mentioned include (1) human-machine interaction and the internet, computing, (2) communication and storage everywhere, (3) the internet of things, (4) artificial intelligence, big data and robotics, (5) the sharing economy and distributed trust, (6) the digitalization of matter to the atomic scale, (7) precise control of molecular biology and nanochemistry, (8) disruptive computing technologies and (9) matter imaging technologies.

Currently, artificial intelligence, big data, and robotics are prominent. Still, I believe that many of the other abovementioned drivers will be essential for the development of chemical sciences in this century. The central thesis of Digital Discovery and its raison d'etre is that these nine drivers with domain sciences such as chemistry, materials science, and biology give birth to a new field at their interface. I like to call this new field "accelerated science", in contrast to the name that the Royal Society of Chemistry gave to this journal. Whatever the future name of this emerging field settles into, its ultimate goal is to reduce the time-todiscovery by reimagining the discovery, optimization and scaleup workflows.

The widely defined fields of computer science and robotics are rapidly transforming the way we live. The early adoption of data science and artificial intelligence (AI) in several areas of industry has led to their digital transformation ${ }^{4}$ but unlike, for example, the cab-driving industry with the rise of global companies such as Uber, Lyft, or 
Didi, the chemical industry is lagging in this journey due to its capital-intensive and plant-centric nature.

The scientific discovery and scaling-up processes can learn from these fields by enabling "self-driving laboratories" or "materials acceleration platforms" that promise to accelerate discovery. ${ }^{5}$ There are new recent demonstrations of such platforms in several areas, and I urge you to check the pages of this journal regularly for even more examples of them.

We welcome forward-looking papers at this large interface of fields and technological drivers. We will consider your article for publication in Digital Discovery if your work is about accelerating discovery or digitizing chemistry, biology, materials science or adjacent fields. We seek innovative, ground-breaking work rather than the routine use of existing methods and incremental advances.

To fully embrace the digital world and open science, Digital Discovery aims to be amongst the first to introduce modern peer review models, data practices, and in general approaches to publication and this is yet another difference from the 1933 journal launch mentioned at the beginning. We begin with having the option of transparent peer review, open access publication, and strong data policies and are working on expanding our content categories to embrace the products of the digital science of this century.

Together with the Editorial Board and the Editorial Office, we are open to your continued suggestions. As scientists in academia, industry, or the government, we can work together to accelerate the scientific process collectively. These (digital) pages should contain the most exciting advances in this emerging field, and I would like to invite you to have a read through them.

\section{References}

1 H. C. Urey, J. Chem. Phys., 1933, 1, 1-2.

2 K. Gavroglu and A. Simoes, Neither Physics nor Chemistry: A History of Quantum Chemistry, MIT Press, 2011.

3 A. Aspuru-Guzik, R. Lindh and M. Reiher, ACS Cent. Sci., 2018, 4, 144152.

4 K. Schwab, The Fourth Industrial Revolution, Crown, 2017.

5 F. Häse, L. M. Roch and A. AspuruGuzik, Trends Chem., 2019, 1, 282-291. 